Piscataway) have isolated and characterized tubulin mutants from Aspergillus, and identified multiple species of tubulins indicating multigenic control of tubulin production and MT polymerization.

Various types of MT can be distinguished by their sensitivity to nocodazole as well as to taxol (M. DeBrabander, Janssen Pharmaceutica Research Laboratories, Beerse). The pattern of assembly and disassembly varies strongly depending on the particular combination of drugs used, indicating different target sites for the two drugs. J. Hyams (University College) showed that methyl benzimidazol carbamate can be used as an inhibitor of MT polymerization in yeast. Thus, mitotic inhibitors for cell types which are less sensitive to the mitotic poisons used in higher plant and animal cells are now available.

W. Cande (University of California, Berkeley) has isolated and characterized a cytoplasmic dynein-like ATPase from sea urchin eggs which binds to different types of MT by bundling them in large parallel arrays, in which many cross-links can be found. The characteristics of the ATPase are consistent with its being a microtubuleassociated protein (MAP) capable of crosslinking MTs in a $\mathrm{Mg}^{2+}$ - and ATP. dependent manner. By careful ultrastructural analyses and a computer-controlled program, C. Jensen (University of Auckland) demonstrated a highly ordered pattern of side arms and cross-bridges. Periodicities of 10-14, 20-24 and $48 \mathrm{~nm}$ are found along parallel MTs. Assuming side arms and cross-bridges represent MAPs, then either MAPs cannot be bound to all tubulin subunits in the MT; or different MAPs may have different binding sites, thus forming the different periodicities.

MAPs can show different patterns of distribution within different cells: whereas MAP2 exhibits a restricted cellular distribution in the brain, MAP1 is widely distributed and prominent in the mitotic spindle (R. Vallee, Worcester Foundation for Experimental Biology, Shrewsbury). Different types of MT can be characterized by their associated MAPs. It is possible that the diversity of MTs is due to their MAPs rather than to their tubulins.

Novel mechanisms for chromosome movement were not proposed. Looking back at all the data presented at the workshop, the attractive notion that one unique mechanism is responsible for the translocation of chromosomes seems no longer tenable. A large body of data has shown that sliding, assembly-disassembly and lateral interaction or zipping (to mention only the three main hypotheses) are all feasible. Clearly a combination of different mechanisms could be responsible for the chromosome transport. The workshop has also shown that we may also have to take into consideration mechanisms which have not yet been discussed at all.

N. Paweletz is Professor at the Institute of Cell and Tumour Biology, German Cancer Research Center, D-6900 Heidelberg 1 .
Geophysics

\section{Magma oceanography and the early evolution of the Earth}

\author{
from Richard W. Carlson
}

A RECENT paper by Anne Hofmeister in Journal of Geophysical Research ${ }^{1}$ presents a model for the evolution of a terrestrial 'magma ocean' created by heat from the impact of accreting planetesimals. Magma ocean models were applied to the Moon almost immediately after the first samples were returned by Apollo 11 (ref. 2) but it is only very recently that similar models have been seriously discussed for the early Earth.

During a planet's formation a large fraction of its energy is transformed to heat in the interior. The heat is lost by solid-state convection but, depending on how efficiently this takes place, additional energy received from the accretion of planetesimals may be sufficient to cause substantial melting. Given an adequate amount of melting, magma will coalesce to form a 'magma ocean' which, in the extreme case, could be of global scale and several hundred kilometres deep. As the magma ocean cools and crystallizes it can produce dramatic physical and chemical effects that will control the future dynamic evolution of the planet.

If the Moon did in fact undergo a magma ocean episode, then the Earth, because of its larger size and hence greater accretional energy, should have melted to an even greater extent. Understanding how a terrestrial magma ocean would behave is complicated by the fact that the phase relationships for the crystallization of silicate magmas at the higher pressures present in the Earth are not available. This limits modelling of a terrestrial magma ocean to a perhaps unrealistically shallow depth of about $120 \mathrm{~km}$. Nevertheless, Hofmeister's work draws some interesting conclusions regarding the minimum size (laterally global), the time required for crystallization $(<10,000 \mathrm{yr}$; a factor of several hundred less than required for the Earth to form from accreting planetesimals) and the crystallization sequence of a $120-\mathrm{km}$ deep terrestrial magma ocean.

To a first approximation, the crystallization sequence of a terrestrial magma ocean that is initially similar in composition to the bulk Earth will resemble that believed to have occurred on the Moon. Put simply, the crystallization produces a layer cake of relatively dense $\mathrm{Mg}$ - and $\mathrm{Fe}$ rich minerals (olivine, pyroxene) overlain by a thick crust of low-density plagioclase (a $\mathrm{Ca}, \mathrm{Al}$ silicate). Plagioclase, being less dense than the magma it crystallizes from, will actually float to the surface of the ocean. These olivine- and plagioclase-rich layers will be convectively stable both with respect to each other and the underlying mantle. In contrast, if the initial composition of the ocean is more similar to a partial melt of the mantle containing more $\mathrm{Ca}$ and $\mathrm{Al}$ than the bulk Earth, the increased pressure gradient of the Earth will allow the early and prolonged crystallization of the dense $\mathrm{Mg}$, Fe, Al silicate, garnet. A major effect of garnet crystallization is that the deep garnet-bearing layers will be denser than the underlying solid mantle and will sink back into it. In this case, only a $30-\mathrm{km}$ thick plagioclase-rich crust will remain as evidence of a magma ocean episode.

In neither of the terrestrial magma ocean models ${ }^{1}$ does this crust actually come close to matching the composition of the crust preserved in the oldest sections of the continents. This failure of a simple shallow magma ocean model to match the geological evidence is compounded further because, unlike rocks on the Moon, rocks of the Earth's crust lack the chemical and isotopic signatures expected to result from an early global molten episode.

While the visible geological evidence for a terrestrial magma ocean is lacking, or at least not obvious, the physical reality of a very hot early Earth is difficult to avoid. Whether this implies that the Earth managed to rid itself of its initial heat without undergoing extensive melting is uncertain. Other possibilities include erasure of the evidence for a magma ocean episode by convective mixing of the crust and mantle over Earth history, or that different and unexpected fractionation paths were caused by the crystallization of unusual high-pressure phases from a deep ocean. Another intriguing paper ${ }^{3}$ notes that because silicate liquids are more compressible than solids, there may be a depth in the mantle, estimated to be about $150 \mathrm{~km}$, where liquids become denser than solids. In this event the terrestrial magma ocean would be confined to great depth and its subsequent evolution would be more difficult to predict.

Whatever the ultimate answer, attempts to understand the evolution of the Earth starting from a given set of initial physical conditions provide a welcome and much needed approach to understanding the early evolution of the solid Earth.

Richard W. Carlson is in the Carnegie Institution of Washington, Department of Terrestrial Magnetism, 5241 Broad Branch Road, NW Washington, DC 20015.

Hofmeister, A.M. J. geophys. Res. 86, 4963 (1983)

2. Wood, J.A., Dickcy, J.S., Marvin U.B. \& Powell, B.N Proc. Apollo 11 Lunar Sci. Conf., 965 (1970).

3. Nisbet, E.G. \& Walker, D. Earth planet. Sci. Lett. 60, 105 (1982). 